Journal of Advanced Computer Science \& Technology, $8(2)(2019) 46-49$
Journal of Advanced Computer Science \& Technology
SPC
Website: www.sciencepubco.com/index.php/JACST
Research paper

\title{
An augmented reality system to enhance facial expressions recognision in autistic children
}

\author{
Hawar Bahzad Ahmad * \\ Department of Computer Science Nawroz University Duhok, Kurdistan Region - Iraq \\ *Corresponding author E-mail: Hawar.doski88@gmail.com
}

\begin{abstract}
In the past decade, Augmented Reality (AR) has been applied in several professional practice areas, including but not limited to autism therapy. AR supplies virtual information to the visual perceptions of users, giving the user new tools to ensure that knowledge for many processes and in many environments are comprehended efficiently. Usually, AR applications depend on virtual reality headset (VR-box) to overlay virtual objects and text on the surrounding environment of the user.

People with autism spectrum condition (ASC) especially children are often found lacking in social interaction, including facial expression and understanding of other minds which make social communication more difficult for them. This paper aims to use AR system to assist children with (ASC) in better understanding facial expressions by overlaying suggested $3 \mathrm{~d}$ objects over the physical environment. Results of AR treatment found higher engagement and more effective compare to a non-AR system.

This paper utilizes Vuforia to create an AR environment for the treatment of children with ASC. Vuforia is a software development kit that provides AR capabilities to smartphone devices.
\end{abstract}

Keywords: Augmented Reality; ASC; Vuforia; Facial Expression.

\section{Introduction}

Augmented Reality (AR) is a technology which enriches the real environment with a certain amount of artificial information; As such the user can see the limitations of the real world being augmented with virtual objects [1]. The perception of facial emotions is significantly affected by the autism spectrum disorder, still little is known about how people with autism spectrum disorder misinterpret facial expressions that have difficulty correctly recognizing facial emotions. The results showed that the difficulty of distinguishing emotional facial expressions from neutral ones accounted by much weaker in the emotional perception of participants with autism spectrum disorder. In particular, adults with autism interpret happy faces in a unique and neutral way [2].

In 2015, studies in the field of teaching strategy showed that children with autism can differentiate between facial expressions but they need more prompting in order to understand the exact emotion to the correct situation [3].There are several methods of therapy for understanding of facial emotions in people with autism or to make it easier for them to understand facial expression better. Exposure therapy is one of the most effective and widely used treatments, one way is where patients are made to watch a special set of films where they are exposed to a wide set of explicit emotion to better enhance their perception of these emotions [4]. However, this process may be difficult for patients due to long duration also some patients cannot focus on television. Second method is using the images as Fig 1 shows. This method is used in many Autism spectrum disorder (ASD) care centers, where individuals with ASD watch pictures of facial emotion and there teacher try to explain the emotion. Another method is AR exposure therapy, which is safe and easy to maintain [5].
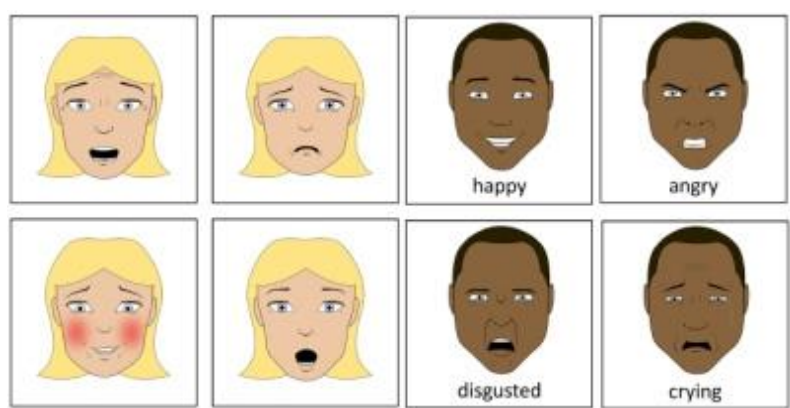

Fig. 1: Emotions Card [6]. 
This research attempts to create a more effective program to treat these kinds of disrders. The hardware requirement to build this AR application is a smart phone and virtual reality VR headset, such as Gear VR [7], which are available with almost all Samsung smart phones. Moreover, Maya3d is used to desiged 3d objects. Also Unity3D is used to create the mobile AR experience. This means that the proposed system is more portable and enables treatment at home. [3]

\section{Related work}

There is a lot of research on understanding ASC social-emotional understanding and how to cure it. However, there is little research on smartphone assisted therapy using AR [8-9]. We are not aware of any AR technique that focuses on ASC social-emotional understanding treatment. But there are some researches on using AR system for pretend play using a computer. Based on the available research, AR systems are designed to teach ASC children how to simulate their imagination [10-11].

\section{Method}

This section presents an Augmented Reality application that aims to encourage facial expression understanding for ASC children. We illustrate the design of an experiment that compares facial expression behaviors between the AR application and an equivalent non-AR setup which is pictures.

\subsection{Implementation}

The system modeling has been done using Autodesk Maya 3d software to create emotional character as shown in Fig 2. Maya 3d software provides a powerful toolset for character creation, motion graphics and animation [12].

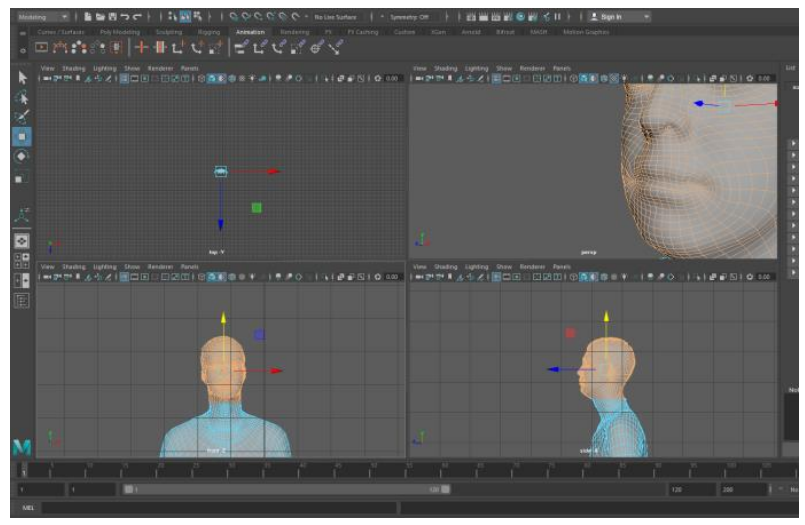

Fig. 2: Modeling A Characters Usig Autodesk Maya.

\subsection{Modelling}

The polygons has been used to modeling six 3d objects "human" with six different basic expressions which are (fear, anger, sadness, happiness, disgust, and surprise) [13-14]. VR mobile phone applications restrict the number of polygons and textures used in the creation of an application [12-20]. However, a large number of polygons were used as in figure 1 and combined together to create one 3D object since Vuforia enables rendering object with large amount of polygons. Also higher number of polygons enables more realistic and accurate design. Here the left side of object been modeled and then copied and reversed using special-duplicate tools to finish modeling faster and more accurate as shown in figure 3.

A) Modeling Left Side of the Face.

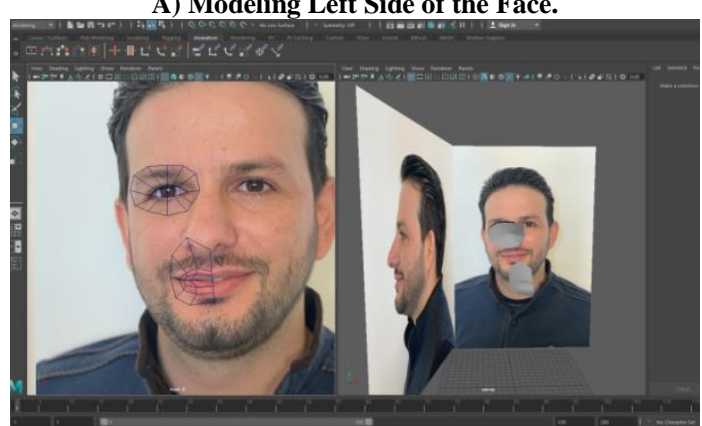

B) Mirroring Left Side of the Face.

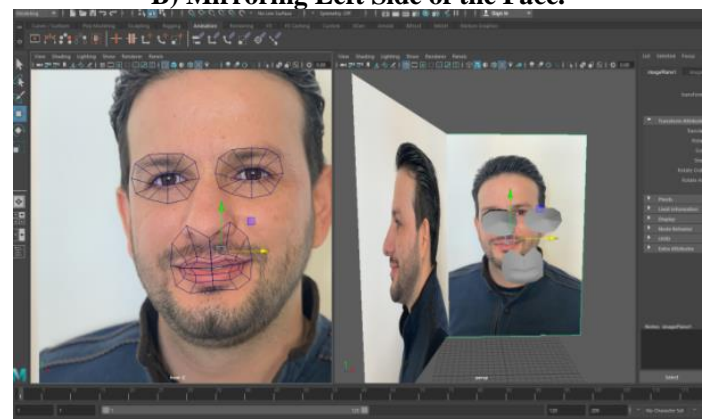

Fig. 3: Modeling Objects.

These objects are textured using various materials such as Lamberts and UV mapping technique for face texturing. See Fig 4. 


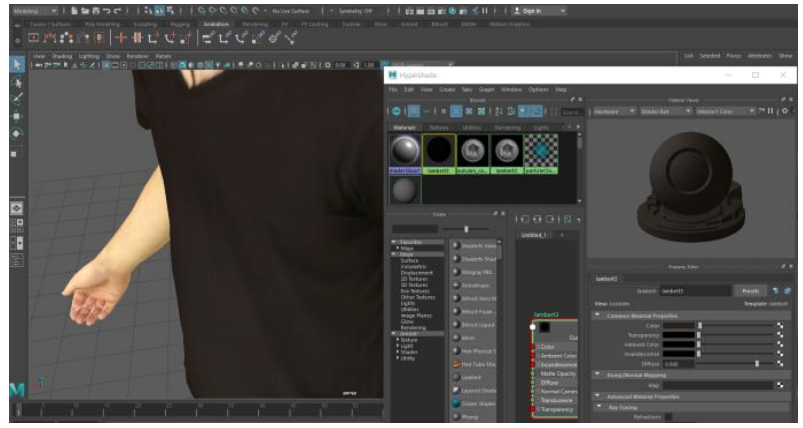

Fig. 4: Texturing Clothes.

\subsection{Scene}

AR Technology has been developed using Unity3d. Unity3d is an engine that enables creating two dimensional and three dimensional, virtual reality and augmented reality applications [15-16]. Moreover, the application is created for smartphones and relies on marker based tracking for facial tracking method. Marker based tracking has been chosen for two reason. First, It is easier for children with autism to interact with the application. Second, it allows a set of objects to be tracked flexibly, Vuforia plugins must be imported into the Unity3d so that it can use Vuforia to build the application [17].

1) Vuforia SDK: Vuforia is an augmented reality software development kit developed by PTC global software company. It support Android, IOS and windows device and also support integration with Oculus SDK for the Gear VR, cardboard Android SDK and Google VR SDK. Vuforia SDK enables target tracking. The concept of target divided into two main classes which are Trackable and TrackableResult. TrackableResult contain a reference to matching a status, targets and pose matrix while Trackable class is defined currently as four subtypes: (Object-Target, Image-Target, Cylinder-Target, and Multi-Target) [18-19]. This research focus on image target which don't need a specific code or black and white image to be recognized .Vuforia uses a series of algorithms to track the presented features into an image. It will render the $3 \mathrm{~d}$ object over the detected target .as for this research the Vuforia API in unity3d game engine will enable tracking of image target from smart phone video camera, calculate orientation and position of the target and render the $3 \mathrm{~d}$ object over the detected image target in real time. Image targets are created on-line via Vuforia portal from JPG or PNG input images. Features extracted from these images are stored in a database and the database is exported as a unity3d package. After importing the database package into unity3d and connected with the images the application will be ready to detect the target image.

2) Scene Implementation: The system is implemented using the personal version of Unity. After, the image and database are imported to unity3d. The objects that were created using Maya3d must be imported and connected with the images. Also, the application configuration must be set for AR/VR capabilities and a special license must be incorporated into the project to enable Vuforia SDK. See Fig 5.

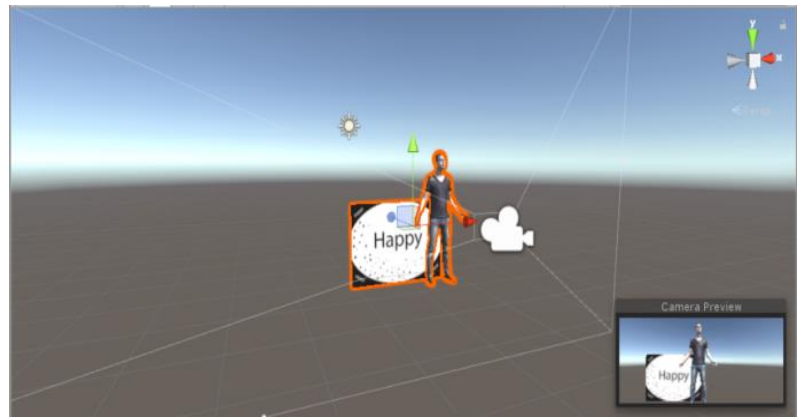

Fig. 5: Image Target and Objects in Unity Editor.

\section{Result}

Results Evidence-based research indicates that AR attracts the attention of autistic children. In fact, many AR studies have been adopted to train children with ASC to imitate facial expressions and emotions to improve their social skills. Example of such treatment are improving autistic children perceptions and judgments of facial expressions and emotions by C. Chien -I. Lee -L. Lin[16] and pretend play for autistic children by Z. Bai, A. F. Blackwell and G. Coulouris [4], etc. After the NAZ-duhok-autism test data were collected for the Autism Care Center, the results showed that AR intervention provided an additional visual indicator that was effectively drawn and drew the attention of children with autism spectrum disorder to nonverbal social signals and helped them better understand facial expressions and the emotions of the characters.

\section{Conclusion}

In conclusion, hardware capabilities in smartphones nowadays enable running high end technology applications such as augmented reality and virtual reality. These technologies can enhance the lifestyle of the disabled, especially children with autism. AR and VR can be put to use to enhance their consciousness and recognition. Specifically tailored applications can be created to suit their different requirement and need, where they can be put on their personal devices for more convenience.

This project created six models of basic human emotions to show the facial expressions and theemotions. This simulation aids young children with ASC in better understanding facial expressions and the emotions of the characters which are more interesting and more attractive compare to emotion cards. 


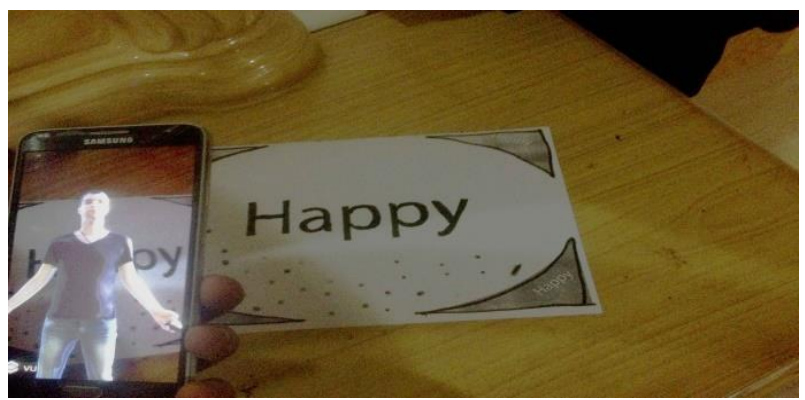

Fig. 6: Character Model at Run Time.

The result has confirmed that autistic children are able to interact with the system.

\section{References}

[1] Denis Kalkofen, Christian Sandor, Sean White, and Dieter Schmalstieg. Visualization Techniques for Augmented Reality. B. Furht (ed.), Handbook of Augmented Reality, (C) Springer Science+Business Media, LLC 2011. https://doi.org/10.1007/978-1-4614-0064-6 3.

[2] Shaun M Eack, Carla A Mazefsky, Nancy J Minshew. Misinterpretation of facial expressions of emotion in verbal adults with autism spectrum disorder. Volume: 19 issue: 3, page(s): 308-315 Article first published online: February 17, 2014; Issue published: University of Pittsburgh, USA. April 1, 2015. https://doi.org/10.1177/1362361314520755.

[3] K. Conallen and P. Reed, "A teaching procedure to help children with autistic spectrum disorder to label emotions", Research in Autism Spectrum Disorders, vol. 23, pp. 63-72, 2016. Available: 10.1016/j.rasd.2015.11.006. https://doi.org/10.1016/j.rasd.2015.11.006.

[4] O. Golan et al., "Enhancing Emotion Recognition in Children with Autism Spectrum Conditions: An Intervention Using Animated Vehicles with Real Emotional Faces", Journal of Autism and Developmental Disorders, vol. 40, no. 3, pp. 269-279, 2009. Available: 10.1007/s10803-009-0862-9 https://doi.org/10.1007/s10803-009-0862-9.

[5] N. S. Lakshmiprabha, A. Santos, D. Mladenov and O. Beltramello, "[Poster] An augmented and virtual reality system for training autistic children," 2014 IEEE International Symposium on Mixed and Augmented Reality (ISMAR), Munich, 2014, pp. 277-278. https://doi.org/10.1109/ISMAR.2014.6948448.

[6] Teaching Aids for Children with Special Needs - Educate Autism", Educateautism.com, $2019 . \quad[O n l i n e] . \quad$ Available: http://www.educateautism.com/. [Accessed: 14- Jan- 2019]. [7] "Oculus Unity Getting Started $\quad$ Guide", Developer.oculus.com, 2019.

[8] Herrera, G., Alcantud, F., Jordan, R., Blanquer, A., Labajo, G. and Pablo C.D. Development of symbolic play through the use of virtual reality tools in children with autistic spectrum disorders. Autism 12, 2 (2008), 143-157. https://doi.org/10.1177/1362361307086657.

[9] Tartaro, A. and Cassell, J. Authorable virtual peers for autism spectrum disorders. In Combined Workshop on ECAI 2006. (8) https://doi.org/10.1145/1240866.1240881.

[10] Z. Bai, A. F. Blackwell and G. Coulouris, "Through the looking glass: Pretend play for children with autism," 2013 IEEE International Symposium on Mixed and Augmented Reality (ISMAR), Adelaide, SA, 2013, pp. 49-58. https://doi.org/10.1109/ISMAR.2013.6671763.

[11] Z. Bai, A. Blackwell and G. Coulouris, "Using Augmented Reality to Elicit Pretend Play for Children with Autism", IEEE Transactions on Visualization and Computer Graphics, vol. 21, no. 5, pp. 598-610, 2015. Available: 10.1109/tvcg.2014.2385092. https://doi.org/10.1109/TVCG.2014.2385092.

[12] A. Hani Alkurdi, "VR Mobile Acrophobia Treatment", Academic Journal of Nawroz University, vol. 6, no. 3, pp. 124-128, 2017. Available: 10.25007/ajnu.v6n3a95. (12)

[13] "Polygonal Modeling | Maya 2016 | Autodesk Knowledge Network", Knowledge.autodesk.com, 2019. [Online]. Available: https://knowledge.autodesk.com/support/maya/learn explore/caas/CloudHelp/

[14] Cloudhelp/2016/ENU/Maya/files/GUID-7941F97A-36E8-47FE-95D1-71412A3B3017-htm. [Accessed: 05- Nov- 2018].

[15] "Unity - Multiplatform - Unity", Unity, 2019. [Online]. Available: https://unity3d.com/unity/features/multiplatform. [Accessed: 02- Jan- 2019].

[16] T. Krogh-Jacobsen, "Introducing Unity 2018.3 - Unity Blog", Unity Technologies Blog, $2019 . \quad$ [Online]. Available: https://blogs.unity3d.com/2018/12/13/introducing-unity-2018-3/. [Accessed: 13- Des- 2018].

[17] P. Ekman, "Are there basic emotions?" Psychological Review, vol. 99, no. 3, pp. 550-553, 1992. Available: 10.1037/0033-295x.99.3.550 [Accessed 14 August 2018]. https://doi.org/10.1037//0033-295X.99.3.550.

[18] "Getting Started with Vuforia Engine in Unity", Library.vuforia.com, $2019 . \quad$ [Online]. Available: https://library.vuforia.com/articles/Training/getting-started-with-vuforia-in-unity.html. [Accessed: 11- Sep- 2018].

[19] "How To Use the Trackable Base Class", Library.vuforia.com, 2019. [Online]. Available: https://library.vuforia.com/articles/Solution/How-ToUse-the-Trackable-Base-Class.html. [Accessed: 17- Sep- 2018].

[20] H. Alkurdi, A. (2019). Augmented Reality Electric Circuit Experiment. 2019 International Conference On Advanced Science And Engineering (ICOASE). https://doi.org/10.1109/ICOASE.2019.8723683. 